Chapter 1

Methods of Design for Micro-Assembly 


\title{
APPLICATION OF A DF $\mu$ A METHODOLOGY TO FACILITATE THE ASSEMBLY OF A MICRO/NANO MEASUREMENT DEVICE
}

\author{
Carsten Tietje', Richard Leach², Michele Turitto', Ronaldo Ronaldo', Svetan \\ Ratchev $^{1}$
}

\author{
'Precision Manufacturing Group, The University of Nottingham, \\ University Park, Nottingham, NG7 2RD, UK. \\ ${ }^{2}$ Industry \& Innovation Division, National Physical Laboratory, \\ Hampton Road, Teddington, TW11 OLW, UK. \\ epxct1@nottingham.ac.uk, richard.leach@npl.co.uk, epxmt2@nottingham.ac.uk, \\ ronaldo.ronaldo@nottingham.ac.uk, svetan.ratchev@nottingham.ac.uk
}

\begin{abstract}
A lack of well defined Design for Microassembly (DF $\mu$ A) methodologies to enable an increased transfer of prototypes from the research lab to production on industrial scale has been identified. The main benefit of such a methodology is the adaptation of the design by matching it with microassembly process characteristics. In addition there needs to be a push in metrology equipment to respond to the ongoing trend of miniaturisation, enabling quality assurance for three dimensional products with nanometer scale features. The presented paper addresses these two gaps by utilising a novel $\mathrm{DF} \mu \mathrm{A}$ methodology to enable a state-of-the-art $\mathrm{CMM}$ stylus assembly, which is characterised by extremely rigid and challenging requirements. The design of the parts to be assembled is shown. Furthermore the selection of the most suitable assembly equipment is supported. Finally the actual assembly system is described and illustrated as proof of validation.
\end{abstract}

\section{Introduction}

The key challenges of manufacturing in a commercial environment can be summarised as delivering products with a competitive price, competitive quality and competitive delivery time. These challenges are supported by a number of production objectives, such as high flexibility, high productivity, constant and high product quality, short throughput times and low production costs [1]. Within the production chain, assembly is a critical step because it forms a substantial part of the total production cost and throughput time. This is particularly true for the microdomain,

Please use the following format when citing this chapter:

Tietje, C., Leach, R., Turitto, M., Ronaldo, R., Ratchev, S,, 2008, in IFIP International Federation for Information Processing, Volume 260, Micro-Assembly Technologies and Applications, eds. Ratchev, S., Koelemeijer, S., (Boston: Springer), pp. 5-12. 
where it is widely accepted that up to $80 \%$ of the production cost of miniaturised systems or hybrid systems occur in assembly $[2,3]$.

The Nexus Market study 2005 predicts the market size for microsystems and microtechnologies to more than double from $€ 16$ billion in 2005 to $€ 36$ billion by the year 2009 [4]. The major market sectors to benefit from the trends of miniaturisation and automation are in the high-technology areas of medical/surgical, automotive and transport, biotechnology and consumer products [5]. To enable these developments it is imperative to develop appropriate manufacturing tools and methods. Under reflection of the above described trends the following two critical needs have been identified and singled out:

- A lack of sufficient Design for Microassembly $(\mathrm{DF} \mu \mathrm{A})$ methodologies to enable an increased transfer of prototypes from the research laboratory to production on industrial scales has been identified. The main benefit is the adaptation of the design by matching it with assembly process characteristics.

- Because "measurement underpins manufacturing technology"[6], there needs to be a push in metrology equipment to respond to the ongoing trend of miniaturisation to enable quality assurance for arising three dimensional products with nanometre scale features.

This paper addresses these two gaps by utilising a novel $\mathrm{DF} \mu \mathrm{A}$ methodology [7] to enable the state-of-the-art coordinate-measuring machine (CMM) stylus assembly, which is characterised by extremely rigid and challenging requirements. A CMM is a programmable, versatile instrument that is used to measure dimensional data for many types of manufactured component. CMMs have three or more measurement axes, usually linear or rotary or a combination of the two. The measurement axes are combined in series so that a unique combination of axes positions defines a single point in space. Measuring an object using a CMM is achieved by moving a measuring probe to a number of points on the object surface in sequence and measuring the position of the probe at each point via the machine scales. The presented paper shows that the DF $\mu \mathrm{A}$ methodology influences the design of the parts to be assembled and furthermore enables the selection of appropriate assembly equipment. The final assembly system and processes and their validation are described and illustrated.

\section{Microassembly and -metrology - scope definition}

\subsection{Critical challenges in microassembly}

"Assembly is defined here as bringing together parts and / or subassemblies, so that a unit comes into being. A subassembly is a composition of parts into a product unit. The assembly process is determined by the manner and the sequence in which the product parts are put together into a complete product. The assembly process comprises a cycle of operations. These operations can be divided into: feeding, 
handling, composing, checking, adjusting and special processes" [1]. Thus it becomes clear that assembly is more than putting parts together. In fact, "Assembly is the capstone process in manufacturing" [8]. Furthermore, assembly is undoubtedly the "least understood process in manufacturing" [8]. Assembly in the microworld faces new challenges hence the complexity increases enormously.

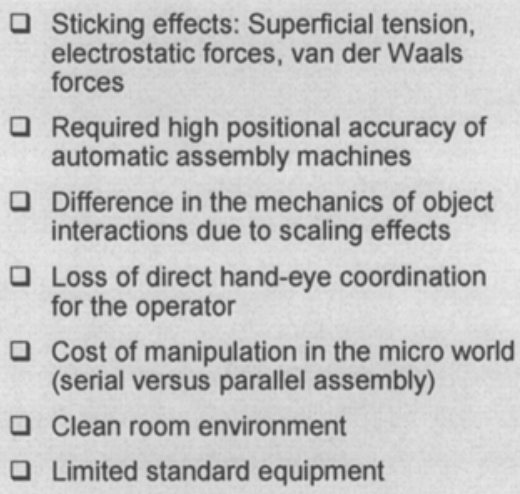

Fig.1. Assembly problems in the micro domain

The main difference between assembly in the macro- and in the microworld is the required positional precision and accuracy. Closed-loop strategies are needed to compensate for poor kinematic models and thermal effects. Another important difference between assembly in the macro- and the microdomain is related to the mechanics of object interactions due to scaling effects [9-11]. In the microworld forces other than gravity dominate. Surface related forces, such as van der Waals, surface tension forces and electrostatic forces become dominant over gravitational forces. Because of this scaling behaviour, manipulation in the microdomain is entirely different from manipulation in the macroworld. As these forces are very difficult to control, they are likely to disturb the assembly process. The object might jump to the gripper and lose orientation or stick to the gripper so that releasing the parts becomes difficult $[9,12]$. An additional problem to sticking effects in the microworld is the loss of direct hand-eye coordination for the operator [9]. Microscopes and tools limit the ability to directly see and sense the objects to be handled.

\subsection{Increasing demand for high accuracy measurement systems}

Most of the devices used for microprocess examination originate from the macroworld and do not fulfil the required demands of microtechnology. Moreover, the downscaling of methods and techniques for quality control from the macroworld is problematic, because experiences and results cannot easily be trans- 
ferred into the microdimension, e.g. resolution, measuring range or image quality restrict the usability [13]. However, the delivery of micro products with nanometre scale features needs to be supported by reliable metrology [6].

Figure 2 gives a schematic overview about micrometrology and identifies four areas, namely material testing, completeness check, dimension and position measurement and function test. The metrological tasks are used mainly for three different components: electronic components, optical components and mechanical components. PFEIFFER ET AL. state that nearly $90 \%$ of the required measurement tasks can be identified as dimension and position measurement [13]. The measurement device the assembly of which is described within this paper will enable better measurements within this significant area, responding to the increasing demand for high accuracy measurement systems.

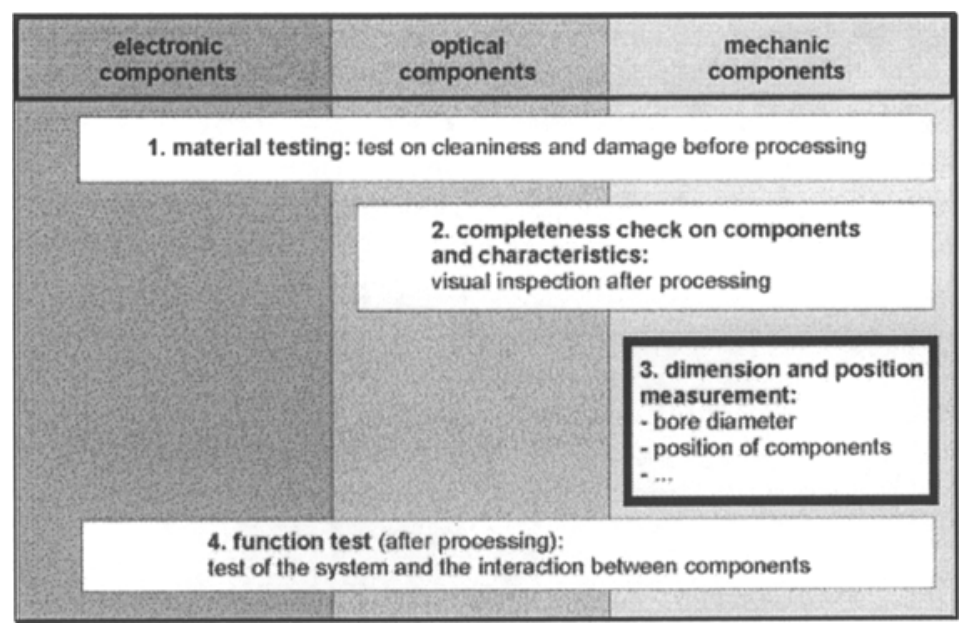

Fig.2. Measurement tasks in the field of microtechnology [13]

\section{Assembly of a micro/nano measurement device}

\subsection{DFuA methodology-conceptual overview}

A novel DF $\mu$ A methodology [7] is used to influence the stylus design and to choose the appropriate assembly processes. The main objective of the methodology is to facilitate improvements in the design process by

- Applying design rules and guidelines which are focused on the microworld to cope with its specific challenges and

- Considering key assembly process features in early design stages. 
Another main objective is to determine the appropriate assembly processes by considering process related requirements. Offering qualitative cost analysis supports the decision making for the assembly system design.

Figure 3 shows the conceptual structure of the DF $\mu \mathrm{A}$ methodology and the underlying models. The first design specifications are based on the product requirements which influence the design the most (mainly functional requirements). Only conceptual drawings are needed. But, like in other DFA methods, the more comprehensive the initial information, the more effective the result. This initial product design are analysed and evaluated by applying the DF $\mu$ A guidelines.

After feeding the input from the $D F \mu A$ guideline model into the design the process-product analysis (which is the key development within the methodology) is carried out. The aim is to consider process related design aspects in the product design and to determine the most appropriate assembly processes. The processes are selected based on the improved design. To decide between several suitable processes a qualitative cost analysis can be conducted to support the decision making.

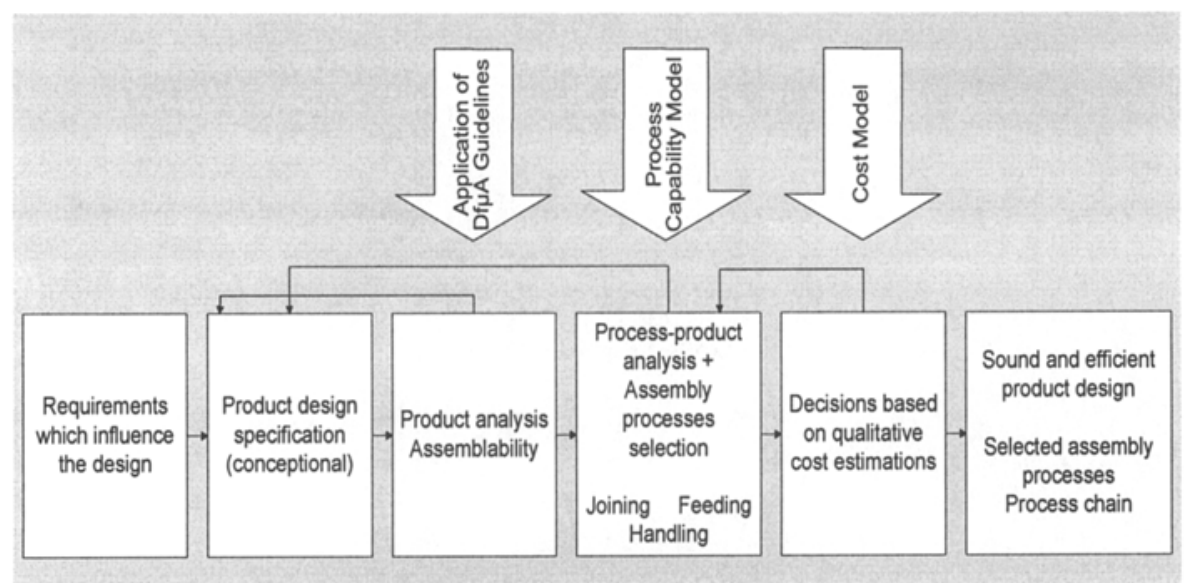

Fig.3. DF $\mu$ A methodology and underlying models [7]

\subsection{Application of the DFMA methodology}

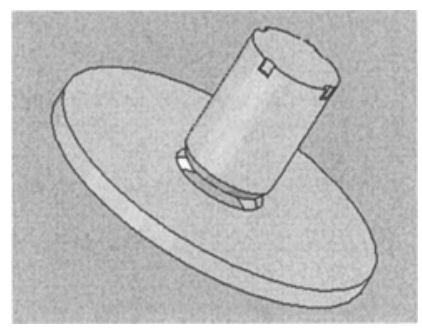

Fig.4. Pin in plate assembly 
Figure 4 presents the assembly of a CMM stylus in a schematic way. To assure functioning of the part it is critical to maintain a $90^{\circ}$ angle between plate and pin. Further essential and challenging tasks such as alignment accuracy, sizes/dimensions, surface roughness, functional elements, environmental constraints (e.g. vibration), reversibility of bonds, volume, part feeding restrictions and fragility, make the assembly of the measurement device unique.

The DF $\mu \mathrm{A}$ methodology is used to influence the stylus design. The figure indicates chamfers on the plate and the pin that aim at easing the assembly and assuring perpendicularity. The four notches in the pin are designed in compliance with the used gripper, guaranteeing the right orientation of the part within the gripper so that the actual insertion process takes place very close to the desired $90^{\circ}$ angle. The chosen appropriate assembly processes are considered in this design. The combination of tight tolerances in the parts design with the high characteristics of the selected assembly processes allows the desired accuracy. Other assembly designs and accordingly assembly process chains were evaluated. The following key points are addressed:

- Match between design and assembly processes (high accuracy positioning, gluing, etc.)

- Relevant design rules (according to requirements)

- Selected processes

- Influenced design features (gripper handle, joint design, capillaries for glue deposition, chamfer etc.)

\subsection{Implementation of the assembly system}

Possible implementations, containing not only the hardware setup and its elements but also relevant validation routes, are described in this section. Figure 5 shows the micro assembly system that is used to assemble the stylus to the plate. The system is characterised by three degrees of freedom realised by three linear piezo-driven stages $(X, Y, Z)$. Furthermore a camera is used to observe the process. A piezodriven gripper, attached in Z-direction to a force sensor is used to manipulate and compose the pin into the plate. A glue dispenser is used to bond the parts together. Figure 5 gives an overview of the whole system, including network controller, light sources as well as an detailed view of the point of work, displaying the linear stages in X- and Y-direction as well as the gripper (together with force sensor attached to linear stage in $\mathrm{Z}$ direction), the camera and the glue dispenser. 

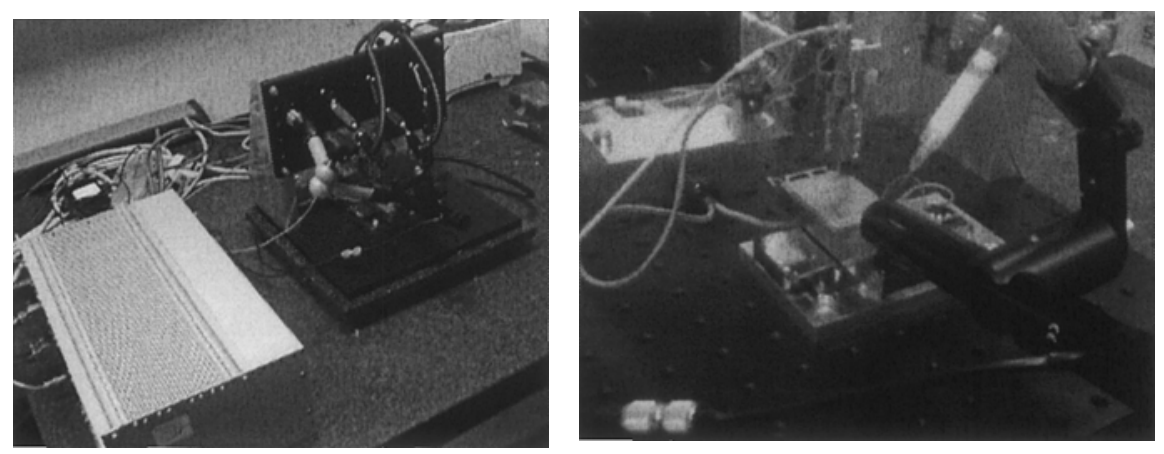

Fig.5. Microassembly station

The chosen system setup allows inspection and supervision of the assembly process. Accurate knowledge on geometry, length, force, pressure and surface roughness of the surveyed parts as well as flow characteristics or the composition of the joining medium (glue) guarantees a high-quality assembly process [13]. The post-assembly evaluation will be extremely challenging and is important to prove the predicted results. For that reason it is envisaged to use the ZEISS F25, for accurate micro/nano metrology. The F25 provides high resolution of $7.8 \mathrm{~nm}$. The tactile sensor is made from the piezoelectric membrane with small probing force at 0.5 $\mathrm{mN}$, and therefore it is non destructive and is ideal for the presented microparts.

\section{Conclusion and outlook}

The assembly process for a novel CMM stylus is described. The design and assembly process selection procedure is supported by a specific DF $\mu \mathrm{A}$ methodology. The presented assembly challenge was used to evaluate the methodology. It can be stated that the preliminary results and the outlined validation are promising but not finally concluded yet. The methodology still relies on human reasoning and interaction but supports the design and process selection process sufficiently. The assembly system layout is described and illustrated and the validation procedure outlined.

Future work can be divided into three strands. The actual validation of the probe assembly needs to be carried out and the assembly needs to be integrated into a metrology system. Finally the DF $\mu \mathrm{A}$ methodology needs to be further developed, in particular the decision making method.

\section{Acknowledgments}

The presented work is part of the ongoing United Kingdom EPSRC "Grand Challenge" research project "3D-Mintegration" which aims to provide radically new ways of thinking for end-to-end design, processing, assembly, packaging, integration and testing of complete 3D miniaturised/integrated '3D Mintegrated' products. The presented assembly challenge is provided by NPL. 


\section{References}

1. Rampersad, H.K., Integrated Simultaneous Design for Robotic Assembly. 1994, Chisester et al.: John Wiley \& Sons.

2. Hesselbach, J. and A. Raatz, eds. mikroPRO, Untersuchung zum internationalen Stand der Mikroproduktionstechnik. 2002, Vulkan: Essen.

3. Koelemeijer, S. and J. Jacot, Cost Efficient Assembly of Microsystems. mst-News, 1999. January: p. 30-33.

4. Unknown, Market Analysis for MEMS and Microsystems III, 2005-2009. 2005: WTC - Wicht Technologie Consulting (on behalf of NEXUS Association).

5. Dimov, S.S., et al. A roadmapping study in $4 M$ Multi-Material Micro Manufacture. in 4M 2006 - SECOND INTERNATIONAL CONFERENCE ON MULTIMATERIAL MICRO MANUFACTURE. 2006 A roadmapping study in 4M MultiMaterial Micro Manufacture. Grenoble: Elsevier (Oxford).

6. Leach, R., et al., Advances in traceable nanometrology at the National Physical Laboratory. Nanotechnology, 2000. 12: p. R1-R6.

7. Tietje, C. and S. Ratchev. Design for Micro Assembly - A methodology for product design and process selection. in IEEE International Symposium on Assembly and Manufacturing (ISAM). 2007. Ann Arbor, USA: Omnipress.

8. Whitney, D.E., Mechanical Assemblies - Their Design, Manufacture, and Role in Product Development. 2004, New York, Oxford: Oxford University Press.

9. Van Brussel, H., et al., Assembly of Microsystems. Annals of the CIRP, 2000. 49(2): p. 451-472.

10. Fearing, R. S. Survey of Sticking Effects for Micro-Parts. in IEEE International Conference for Robotics and Intelligent Systems IROS '95. 1995. Pittsburgh.

11. Ando, Y., H. Ogawa, and Y. Ishikawa. Estimation of attractive force between approached surfaces. in Second Int. Symp. on Micro Machine and Human Science. 1991. Nagoya, Japan.

12. Tichem, M., D. Lang, and B. Karpuschewski, A classification scheme for quantitative analysis of micro-grip principles. Assembly Automation, 2004. 24(1): p. 8893.

13. Pfeifer, T., et al., Quality control and process observation for the micro assembly process. Measurement. 30(1): p. 1-18. 